\title{
CONSUMO ALIMENTAR E ESTADO NUTRICIONAL DE MULHERES COM CÂNCER DE MAMA EM TRATAMENTO QUIMIOTERÁPICO
}

\author{
Isabela de Moraes Pereira, Isabella da Silva Pardim, Sandra Cristina Genaro \\ Universidade do Oeste Paulista - UNOESTE, Presidente Prudente, SP. E-mail: izabelinhamoraes@hotmail.com
}

\section{RESUMO}

Objetivou-se analisar o consumo alimentar e o estado nutricional de mulheres com câncer de mama em tratamento quimioterápico. A amostra foi de 14 mulheres, acima de 19 anos, sobreviventes de câncer de mama em tratamento quimioterápico do Centro Prudentino de Oncologia, da cidade de Presidente Prudente, SP. Destas, 64,3\% estavam acima do peso; 71,43\% apresentaram deficiente consumo de vitamina A, vitamina $E(78,57 \%)$ e $71 \%$ possuíam consumo inadequado de fibras. Observou-se consumo de $24 \pm 9,6$ $\mathrm{kcal} / \mathrm{kg} / \mathrm{P}$ e 0,7士0,4 g/kg de proteína. Conclui-se que o consumo alimentar está aquém de ser adequado e o excesso de peso ainda é prevalente nesse grupo de mulheres. É de extrema importância a elaboração de estratégias de reeducação alimentar, salientando a relevância da alimentação saudável e equilibrada para prevenção e tratamento de doenças, inclusive o câncer de mama, visando a melhora do estado nutricional, manutenção do peso saudável, contribuindo dessa forma para um bom prognóstico.

Palavras-chaves: Neoplasia Maligna da Mama. Alimentação. Fatores de Risco. Obesidade. Quimioterapia. Avaliação nutricional.

\section{DIETARY INTAKE AND NUTRITIONAL STATUS OF WOMEN WITH BREAST CANCER UNDERGOING CHEMOTHERAPY}

\begin{abstract}
The objective of this study was to analyze the food intake and nutritional status of women with breast cancer undergoing chemotherapy. The sample consisted of 14 women, over 19 years old, survivors of breast cancer undergoing chemotherapy treatment at the Prudentino Center of Oncology, in the city of Presidente Prudente, SP. Of these, $64.3 \%$ were overweight; $71.43 \%$ had deficient intake of vitamin $A$, vitamin $\mathrm{E}(78.57 \%)$ and $71 \%$ had inadequate fiber intake. Consumption of $24 \pm 9.6 \mathrm{kcal} / \mathrm{kg} / \mathrm{P}$ and $0.7 \pm 0.4$ $\mathrm{g} / \mathrm{kg}$ of protein was observed. It is concluded that food intake is below adequate and overweight is still prevalent in this group of women. It is extremely important to develop strategies for dietary reeducation, emphasizing the importance of healthy and balanced diet for the prevention and treatment of diseases, including breast cancer, aiming at improving nutritional status, maintaining healthy weight, thus contributing to a good prognosis.
\end{abstract}

Keywords: Malignant Breast Neoplasia. Food. Risk factors. Obesity. Chemotherapy. Nutritional assessment.

\section{INTRODUÇÃO}

Responsável por uma das maiores causas de mortalidade tanto no Brasil como no mundo, o câncer é considerado um evidente problema de saúde pública ${ }^{1}$. Suas causas primárias ainda não estão totalmente esclarecidas, porém sabe-se que as neoplasias malignas surgem devido às Colloq Vitae 2020 set-dez; 12(3): 26-36.

Artigo Open Access sob uma licença CC BY-NC-ND (http://creativecommons.org/licenses/by-nc-nd/4.0/). mutações genéticas espontâneas, ou induzidas por agentes patogênicos como metais, radiações, radicais livres do oxigênio, inflamações crônicas e xenobióticos (tabaco, álcool, pesticidas, etc.), os quais promovem desordem no ciclo celular, ocorrendo excesso na taxa de proliferação e deficiência na apoptose celular². 
Dentre os tipos de câncer existentes, 0 câncer de mama teve um aumento de sua incidência tanto no Brasil $^{3}$, como em países subdesenvolvidos e desenvolvidos ${ }^{4}$, apesar do aumento de campanhas para o diagnóstico precoce. Sendo considerado a neoplasia mais prevalente em mulheres, estima-se que para o biênio 2018/2019 o Brasil apresente 59.700 novos casos de câncer de mama feminino ${ }^{5}$.

Em geral, o primeiro sinal da doença costuma ser a presença de um nódulo único, não doloroso e endurecido, apresentando deformidades e aumento da mama, podendo ocorrer retração da pele do mamilo, onde os gânglios axilares apresentam-se aumentados, causando vermelhidão, edema, dor e muitas vezes presença de líquidos ${ }^{6}$.

O câncer de mama não possui uma causa aparente. Geralmente é mais comum em mulheres após os 50 anos de idade, porém, diversos fatores estão relacionados ao aumento do risco de desenvolver a doença, dentre eles os fatores genéticos e hereditários; hormonais; comportamentais e ambientais ${ }^{7}$.

Um dos fatores que levam a esse tipo de câncer são mutações genéticas familiares (genes BRCA1 e BRCA2); história de casos de câncer de mama em parentes próximos, principalmente em idade jovem; histórico na família de câncer de ovário ou câncer de mama em homem ${ }^{7-9}$, outro fator é a exposição prolongada ao hormônio estrogênio produzido pelo próprio organismo; reposição hormonal por longo período; menarca precoce (menos de 12 anos); menopausa tardia (após 55 anos); mulheres que não tiveram filhos ou tiveram o primeiro após os 35 anos; mulheres que não amamentaram? ${ }^{7}$.

A obesidade, principal consequência do consumo excessivo de alimentos calóricos como açúcares, gorduras e bebidas alcoólicas, é responsável pelo aumento da gordura visceral e circunferência da cintura maior que $80 \mathrm{~cm}$ em mulheres, a qual parece influenciar no desenvolvimento e na progressão do câncer de mama ${ }^{10-12}$. Pesquisas têm demonstrado que o excesso de peso após a menopausa, incluindo um consumo de alimentos com alto teor calórico, dieta rica em proteínas e gordura animal, excesso de carne vermelha, bebida alcoólica e carnes processadas são responsáveis para 0 desenvolvimento desse tipo de câncer ${ }^{8,9,13}$.

O excesso de peso leva a uma maior exposição do epitélio mamário à várias substâncias produzidas pelo tecido adiposo ${ }^{14}$, Colloq Vitae 2020 set-dez; 12(3): 26-36.

Artigo Open Access sob uma licença CC BY-NC-ND (http://creativecommons.org/licenses/by-nc-nd/4.0/). dentre elas, o estrogênio. Seu excesso é responsável por causar resistência periférica à insulina, elevando os níveis do fator de crescimento dependente de insulina (IGF-1), dos quais participam da tumorigênese mamária ${ }^{15}$. Além disso, o excesso de gordura corporal, por ter relação com processos inflamatórios, aumentam citocinas como fator de necrose tumoral- $\alpha$ e fator de crescimento endotelial vascular (VEGF), envolvidos na regulação da angiogênese ${ }^{14,16}$.

Embora o câncer de mama possua diversos fatores, dentre eles os não modificáveis, sua prevenção é baseada nos fatores modificáveis $^{17}$, como a obesidade ${ }^{9,17}$. Com melhora no estilo de vida através da prática de atividade física regular e alimentação balanceada, com ênfase na ingestão de frutas, verduras e legumes ${ }^{18}$, evitando o consumo de alimentos ditos como fatores de risco ${ }^{9}$, é possível reduzir em até $28 \%$ a formação do câncer de mama ${ }^{17}$. Tendo como alimentação saudável, a ingestão de alguns componentes alimentares capazes de auxiliar nessa prevenção, como fibras; vitaminas e minerais (presentes em frutas, verduras e legumes); alimentos fitoquímicos (isoflavonas, lignanas); ácidos graxos poli-insaturados (ômega 3) e ácido linoleico conjugado (CLA) ${ }^{11}$.

O tratamento para o câncer de mama pode ser dividido em loco-regional, por radioterapia e cirurgia; e sistêmico, através da hormonioterapia e quimioterapia ${ }^{19}$, utilizando diversos protocolos.

É comum encontrarmos mulheres em tratamento sistêmico apresentando um índice de massa corpórea (IMC) superior ao de mulheres com diagnóstico recente da doença, devido à inatividade física e aos medicamentos utilizados na quimioterapia ${ }^{20}$, os quais influenciam no aumento do apetite, levando a uma redução da qualidade alimentar ${ }^{10,11}$.

Sabe-se que o excesso de peso está ligado a um pior desfecho no prognóstico de mulheres que possuem ou que já tiveram câncer de mama ${ }^{21}$, como descreve uma revisão sistemática entre os anos de 1990 e 2001, observando que 29.460 pacientes apresentaram relação com a obesidade e a recorrência do câncer de mama com aumento da mortalidade ${ }^{22}$.

Este trabalho teve como objetivo, analisar o consumo alimentar e o estado nutricional de mulheres com câncer de mama em tratamento quimioterápico para orientá-las a uma alimentação adequada, visando melhorar o 
prognóstico e a qualidade de vida dessas pacientes, prevenindo recidivas relacionadas aos fatores modificáveis.

\section{MÉTODOS}

A pesquisa foi iniciada após a aprovação do Comitê de Ética em Pesquisa através do protocolo 08701019.3.0000.5515.

Tratou-se de um estudo observacional, descritivo com delineamento transversal de análise qualitativa e quantitativa, realizado no Centro Prudentino de Oncologia (CPO), da cidade de Presidente Prudente, SP. A proposta amostral seria de 30 pacientes do sexo feminino, acima de 19 anos, com diagnóstico de câncer de mama, em tratamento quimioterápico. Esse número de pacientes condiz com o número de mulheres que fazem tratamento para câncer de mama no referido centro. As pacientes que aceitaram o convite, assinaram um termo de consentimento livre e esclarecido (TCLE) redigido para este fim, no qual foram informadas sobre os objetivos do trabalho e os procedimentos aos quais foram expostas.

Foi aplicado um questionário sociodemográfico, elaborado pelas próprias pesquisadoras, com intuito de conhecer o perfil das pacientes do estudo. Além disso, as mulheres foram questionadas sobre os fatores de risco para desenvolvimento do câncer de mama como uso de terapia de reposição hormonal, sedentarismo (ausência de prática e frequência de exercícios físicos regulares, considerando no estudo mulheres com atividade física com frequência mínima de três vezes por semana e duração não inferior a 30 minutos), consumo regular de bebidas alcoólicas e tabagismo antes do diagnóstico, prática de dietas alimentares restritivas e questionamento quanto ao peso usual antes do diagnóstico da doença.

Para classificação do estado nutricional, foram utilizados Índice de Massa Corporal (IMC), Dobra Cutânea Triciptal (DCT), Circunferência do Braço (CB), Circunferência Muscular do Braço $(\mathrm{CMB})$, Área Muscular do Braço Corrigida (AMBC).

Utilizou-se os critérios da World Health Organization $^{23}$ para adultos; e Lipschitz ${ }^{24}$ para idosos ( $\geq 60$ anos) para classificação do índice de massa corporal (IMC).

A Circunferência do Braço (CB) foi obtida com a paciente em pé, com o braço esquerdo flexionado em 90 graus e com o auxílio de uma fita métrica inelástica flexível, de $150 \mathrm{~cm}$ e variação de $1 \mathrm{~mm}$, o qual foi aferido o ponto Colloq Vitae 2020 set-dez; 12(3): 26-36.

Artigo Open Access sob uma licença CC BY-NC-ND (http://creativecommons.org/licenses/by-nc-nd/4.0/) médio entre o acrômio que fica localizado entre a clavícula e o úmero, e o olecrano que é localizado entre o úmero e a ulna. Após, com o braço estendido ao longo do corpo com a palma da mão voltada para a coxa, foi contornado o braço com a fita métrica no ponto médio marcado de forma ajustada, evitando-se a compressão da pele ou folga ${ }^{25}$.

Ainda com a paciente em pé, a Dobra Cutânea Triciptal (DCT) foi aferida na face posterior do braço, paralelamente ao eixo longitudinal, no ponto que compreende a metade da distância entre a borda súperolateral do acrômio e o olecrano ${ }^{26}$. Os resultados foram avaliados segundo os critérios estabelecidos na literatura considerando-se depleção para P15 e excesso de gordura para $\mathrm{P}^{2} 5^{27}$.

Tanto para obtenção da CB como da DCT, foram utilizados o braço contrário à mama doente para evitar erros devido a possíveis edemas.

Foi calculado a CMB através da equação: [CB $(\mathrm{cm})-(0,314 \times$ DCT $(\mathrm{mm})]$; e a AMBc, através da equação para mulheres: $\left[(\mathrm{CMB})^{2} \div 12,56\right]-$ 6,51925.

Para avaliação do consumo alimentar, foi realizado o "Registro de Consumo Alimentar" (RCA) de 3 dias alternados (no início, meio e final da semana). Através de um Software on line, de Nutrição, foi possível obter a quantidade de calorias, macronutrientes e antioxidantes como vitamina A, C, E; selênio e fibras dos registros alimentares. Em seguida obteve-se a média dos valores calculados e foi comparado com as Dietary reference intakes (DRI) ${ }^{28}$.

Para a análise dos dados foi criado um banco de dados e as informações foram expressas de forma quantitativa, apresentados de forma descritiva expressos através de tabelas, gráficos a partir do programa Microsoft Office Excel $^{\circledR}$.

\section{RESULTADOS}

Da proposta de serem avaliadas 30 mulheres com câncer de mama acima de 19 anos, apenas 14 pacientes participaram da pesquisa, devido a muitas intercorrências ocorridas, como indisposições das pacientes, alterações nos horários da quimioterapia, recusa em participar da pesquisa e um óbito.

As mulheres que concordaram em participar do estudo apresentaram as seguintes características: a média de idade das pacientes era de $53,79 \pm 19,97$ anos, no qual $85,71 \%(n=12)$ 
pertenciam à etnia branca e $14,28 \%(n=2)$ à etnia parda. Dentre elas, $71,43 \%(n=10)$ possuíam ensino superior completo; $7,14 \%(n=1)$, segundo grau incompleto; $14,28 \%(n=2)$, segundo grau completo e $7,14 \%(n=1)$, primeiro grau completo.

A prevalência do estado civil $71,43 \% \quad(n=10)$ casadas, $21,43 \%(n=3)$ solteiras e $7,14 \%(n=1)$ possuía união estável.

Tabela 1. Fatores de risco para câncer de mama das mulheres do estudo

\begin{tabular}{lrr}
\hline Variáveis & \% & $\mathbf{n}$ \\
\hline Menarca precoce (<12 anos) & $7,14 \%$ & 1 \\
\hline Menopausa tardia (>55 anos) & $7,14 \%$ & 1 \\
\hline Nuliparidade & $42,85 \%$ & 6 \\
\hline Uso de Terapia de Reposição Hormonal & $14,28 \%$ & 2 \\
\hline Tabagismo & $14,28 \%$ & 2 \\
\hline Consumo de Bebida Alcoólica & $0 \%$ & 0 \\
\hline Obesidade ou Sobrepeso & $42,85 \%$ & 6 \\
\hline Sedentarismo & $28,57 \%$ & 4 \\
\hline
\end{tabular}

A tabela 2 apresenta os resultados das variáveis antropométricas que foram utilizadas para obtenção do diagnóstico nutricional e a
A Tabela 1 apresenta as características das mulheres do estudo, quanto aos fatores de risco para câncer de mama, antes de desenvolverem a doença. tabela 3, apresenta o diagnóstico nutricional atual das mulheres do estudo. 
Tabela 2. Resultado das variáveis antropométricas utilizadas

\begin{tabular}{|c|c|c|c|c|c|c|}
\hline \multirow{2}{*}{ Paciente } & \multirow{2}{*}{ Idade } & \multirow{2}{*}{ IMC } & \multirow{2}{*}{$\begin{array}{c}\text { Adequação } \\
\text { CB (\%) }\end{array}$} & \multirow{2}{*}{$\begin{array}{r}\text { Adequação } \\
\text { DCT (\%) }\end{array}$} & \multirow{2}{*}{$\begin{array}{r}\text { Adequação } \\
\text { CMB (\%) }\end{array}$} & \multirow{2}{*}{$\begin{array}{c}\text { Adequação } \\
\text { AMBc (\%) }\end{array}$} \\
\hline & & & & & & \\
\hline 1 & 54 & 25 & 98 & 100 & 100 & entre P25 e P50 \\
\hline 2 & 53 & 29 & 101 & 118 & 98 & entre P25 e P50 \\
\hline 3 & 64 & 23 & 92 & 85 & 95 & entre $\mathrm{P} 25$ e $\mathrm{P} 50$ \\
\hline 4 & 64 & 19 & 75 & 69 & 77 & $<\mathrm{P} 5$ \\
\hline 5 & 46 & 29 & 113 & 176 & 90 & entre P15 e P25 \\
\hline 6 & 71 & 31 & 109 & 108 & 79 & $<$ P5 \\
\hline 7 & 36 & 20 & 78 & 111 & 65 & $<$ P5 \\
\hline 8 & 60 & 21 & 86 & 96 & 83 & entre $\mathrm{P} 15$ e $\mathrm{P} 25$ \\
\hline 9 & 67 & 35 & 115 & 132 & 109 & entre P85 e P90 \\
\hline 10 & 56 & 30 & 104 & 115 & 100 & entre P50 e P75 \\
\hline 11 & 44 & 30 & 108 & 163 & 89 & entre $\mathrm{P} 25$ e P50 \\
\hline 12 & 53 & 29 & 106 & 165 & 88 & entre P25 e P50 \\
\hline 13 & 50 & 36 & 128 & 149 & 125 & $>$ P95 \\
\hline 14 & 35 & 27 & 124 & 183 & 105 & entre P75 e P85 \\
\hline
\end{tabular}

Tabela 3. Estado nutricional atual das mulheres do estudo.

\begin{tabular}{lcc}
\hline Estado Nutricional & $\mathbf{6}$ & $\mathbf{n = 1 4}$ \\
\hline Desnutrição proteico calórica leve & 7,14 & 1 \\
Desnutrição proteico calórica moderada & 7,14 & 1 \\
\hline Eutrofia & 7,14 & 1 \\
Eutrofia com depleção discreta de tecido adiposo & 7,14 & 1 \\
Eutrofia com depleção muscular moderada & 7,14 & 1 \\
\hline Sobrepeso & 35,71 & 5 \\
Sobrepeso com depleção muscular leve & 7,14 & 1 \\
Sobrepeso com depleção muscular moderada & 7,14 & 1 \\
\hline Obesidade leve com depleção muscular leve & 7,14 & 1 \\
Obesidade moderada & 7,14 & 1 \\
\hline
\end{tabular}

A tabela 4 apresenta a análise dos dados obtidos no registro alimentar de acordo com as variáveis analisadas: energia total estimada por $\mathrm{kg}$ de peso, carboidratos por $\mathrm{kg}$ de peso, proteínas por $\mathrm{kg}$ de peso, lipídios por $\mathrm{kg}$ de peso e suas respectivas porcentagens em relação ao valor calórico total.
O recordatório de registro alimentar das mulheres com câncer de mama em tratamento quimioterápico, referente às concentrações de Vitaminas A, C, E e o mineral Selênio, foram comparados com as recomendações diárias $(D R I)^{28}$ e demonstrados na figura 1.

Tabela 4. Valores das médias e desvios padrão dos dados obtidos no registro alimentar.

\begin{tabular}{lr}
\hline \multicolumn{2}{c}{ Variáveis } \\
\hline Calorias totais & $\mathbf{n = 1 4}$ \\
Calorias totais/kg/P & $1569 \pm 442,8$ \\
CHO g/kg & $24 \pm 9,6$ \\
CHO \% & $3,2 \pm 1,3$ \\
PTN g/kg/P & $53 \pm 6,3$ \\
PTN \% & $0,7 \pm 0,4$ \\
LIP g/kg/P & $20,5 \pm 4,5$ \\
LIP \% & $0,7 \pm 0,4$ \\
& $27 \pm 5,8$ \\
\hline
\end{tabular}




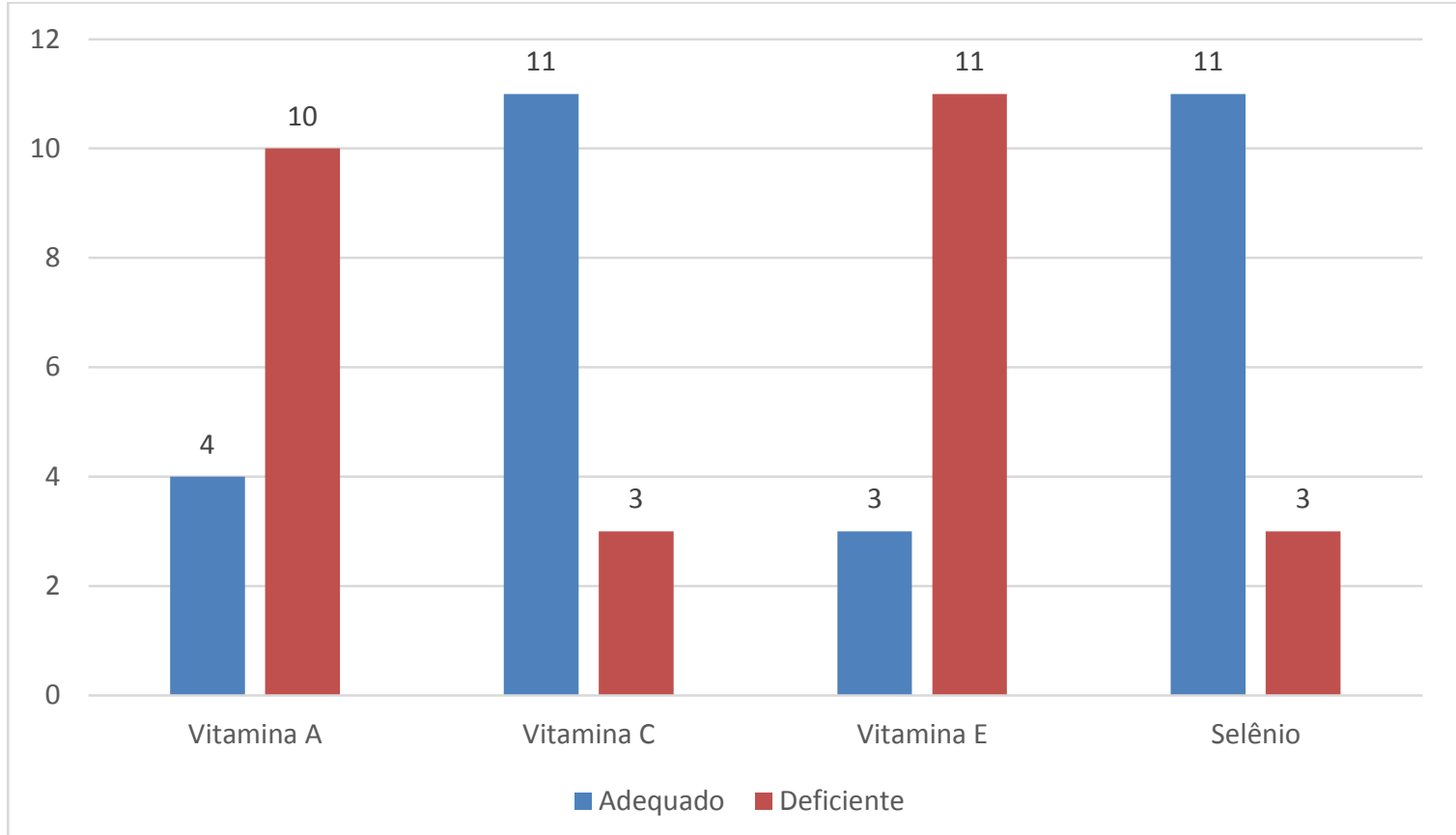

Figura 1. Comparação do RCA e DRI de Vitaminas A, C, E e Selênio das mulheres do estudo.

A maioria das mulheres $(71,43 \%)$ apresentou uma média deficiente no consumo de vitamina A e de vitamina $\mathrm{E}(78,57 \%)$; e consumo adequado em vitamina $C(78,57 \%)$ e selênio (78,57\%), sendo que a média do consumo de Vitaminas A estava entre $412 \pm 237 \mathrm{mcg}$, vitamina C $52 \pm 36 \mathrm{mg}$, vitamina E $9 \pm 3 \mathrm{mg}$ e $31 \pm 19 \mathrm{mcg}$ de selênio.
O consumo de fibras apresentou-se abaixo do recomendado, $71 \%$ das mulheres relataram consumir uma média de $14 \pm 3 \mathrm{~g}$ de fibras ao dia e somente $29 \%$ apresentaram consumo adequado, ou seja, acima de $21 \mathrm{~g}$ por dia, preconizado pela $D R I^{28}$, como ilustra a figura 2.

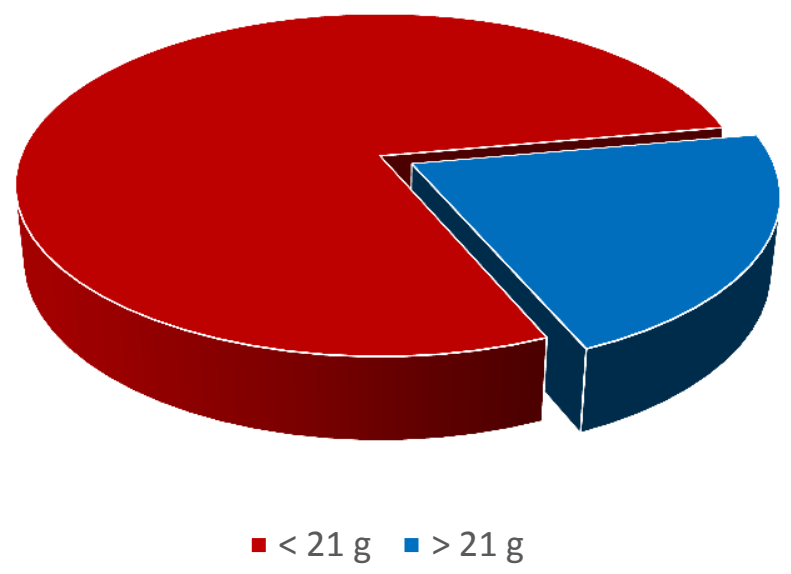

Figura 2. Mulheres com câncer de mama em tratamento quimioterápico e seu consumo de fibras.

\section{DISCUSSÃO}

São diversos fatores de risco que contribuem para o desenvolvimento do câncer de mama como raça, estilo de vida e casos na família, entretanto, a obesidade ou excesso de peso tem mostrado cada vez mais associação, apresentando uma relação estreita entre o acúmulo de gordura abdominal e o câncer de 
mama. A manutenção do peso nessas mulheres acaba não sendo tão simples, devido ao fato de alguns quimioterápicos contribuírem para 0 ganho de peso pelo aumento do apetite ${ }^{29}$, associado à inatividade física ${ }^{10,11}$ e também retenção hídrica ${ }^{30}$.

A obesidade também é um fator de mal prognóstico e de recidiva do câncer de mama em mulheres que têm ou tiveram essa doença, principalmente no período pós menopausa. Por causar um estado pró-inflamatório, a obesidade libera mediadores inflamatórios propiciando o crescimento tumoral, além de acarretar desenvolvimento de outras patologias, como diabetes e doenças cardiovasculares, contribuindo para a morbimortalidade ${ }^{31,32}$. O presente trabalho apresentou algum grau de obesidade em $64,3 \%$ das mulheres, assim como em um estudo realizado por Rubin et $\mathrm{al}^{33}$, o qual observou que $57,14 \%$ das mulheres adultas e $63,49 \%$ das mulheres idosas com câncer de mama em tratamento quimioterápico estavam acima do peso. Em outro estudo feito por Lagares et $\mathrm{al}^{34}$, essa porcentagem é ainda superior, sendo $87,5 \%$ para excesso de peso ${ }^{34}$.

A alimentação tem um importante papel no tratamento de doenças, incluindo $o$ tratamento do câncer, devendo ser equilibrada, seguindo as recomendações das diretrizes e consensos.

No presente estudo, observamos que a média das porcentagens de macronutrientes estavam de acordo com Dietary reference intakes $(D R I)^{28}$. Considerando a média por $\mathrm{kg}$ de peso, encontramos valores adequados de calorias $(24 \pm 9,6 \mathrm{kcal} / \mathrm{kg} / \mathrm{P})$ quando comparadas com o Consenso de Nutrição Oncológica ${ }^{35}$ para pacientes adultos obesos em tratamento clínico (quimioterapia e radioterapia), ou seja, de 20 a $25 \mathrm{kcal} / \mathrm{kg}$. Todavia, dentre as entrevistadas $(100 \%), 21,43 \% \quad(n=3)$ encontravam-se em manutenção de peso e $14,3 \% \quad(n=2)$ apresentavam repleção de peso, discordando das recomendações para pacientes obesos, em que a média de kcal por kg deveria ser de 25 a 30 $\mathrm{kcal} / \mathrm{kg}$ e 30 a $35 \mathrm{kcal} / \mathrm{kg}$ respectivamente.

Para as necessidades proteicas diárias, observamos um valor abaixo do recomendado $(0,7 \pm 0,4 \mathrm{~g} / \mathrm{kg})$ pelo Consenso de Nutrição Oncológica, o qual recomenda ingestão diária de proteína de $1,0 \mathrm{~g} / \mathrm{kg}$ a $2,0 \mathrm{~g} / \mathrm{kg}$, dependendo do grau de estresse e da depleção de massa muscular. Os valores encontrados no presente trabalho, discordam do estudo realizado por
Cattafesta et al. $^{36}$, os quais observaram que o consumo de proteínas em mulheres no tratamento de câncer de mama encontravam-se adequado, porém com um maior consumo de kcal.

Pacientes oncológicos podem apresentar deficiências de micronutrientes em função do aumento das necessidades e de perdas associadas à diminuição da ingestão alimentar ${ }^{37}$ bem como aos efeitos do tratamento, os quais são responsáveis por alterações na absorção, anorexia, desidratação, vômitos e diarreia ${ }^{38}$.

O consumo de alimentos que possuem substâncias antioxidantes como frutas, legumes e verduras; bem como a prática regular de atividade física têm sido relacionados à diminuição da incidência de doenças crônicas degenerativas; assim como o tratamento dietoterápico utilizando alimentos ricos em antioxidantes tem se mostrado eficaz, principalmente em patologias com alto estresse oxidativo, como o câncer ${ }^{39}$. A obesidade, quadro em que grande parte das mulheres deste trabalho se encontram, também é responsável por promover o aumento do estresse oxidativo proporcionado pelas citocinas inflamatórias, o que juntamente com a má ingestão de alimentos antioxidantes, levam ao aumento de radicais livres $^{31}$.

De acordo com o Consenso Nacional De Nutrição Oncológica de $2016^{38}$, os antioxidantes são capazes de minimizar os efeitos da quimioterapia, principalmente quando se fala de desconforto gastrointestinal.

Os carotenoides, pigmentos naturais encontrados em frutas e vegetais ${ }^{40}$, possuem um papel fundamental na prevenção e tratamento do câncer devido ao seu potencial antioxidante, capaz de eliminar o radical peróxido e capturar o radical peroxila. Dentre eles, o licopeno tem um papel importante na proteção de moléculas como as lipoproteínas e DNA.

A vitamina $C$, encontrada em alimentos como vegetais folhosos de coloração verde escuro e frutas cítricas, também desempenha importante papel na prevenção da carcinogênese, impedindo seu processo pelos mecanismos de alteração dos carcinógenos.

A vitamina $E$ por sua vez, promove a desaceleração do crescimento das células malignas devido seu potencial em gerar a inibição da peroxidação lipídica e proteção das membranas. Não somente isso, essa vitamina possui propriedades anti-inflamatória e 
antitrombótica, podendo ser encontrada principalmente em oleaginosas e grãos integrais.

O selênio (mineral) pode ser encontrado em diversos alimentos, tanto de origem animal (carnes, leite e derivados) como de origem vegetal (repolho, couve flor, brócolis, cebola, mostarda da Índia, castanha do Pará). Assim como os demais antioxidantes, o selênio influencia no processo da carcinogênese devido a proteção pelas selenoproteínas, as quais protegem as células dos radicais livres ${ }^{38}$, evitando também o processo de apoptose celular ${ }^{39}$.

Nos últimos anos, a ingestão de fibras pela população diminuiu consideravelmente, contrapondo-se com a obesidade. As fibras, tanto solúveis como insolúveis, possuem variadas funções como controle de diarreia, prevenção da constipação intestinal, redução da absorção de carboidratos, retardo do esvaziamento gástrico, prevenção da obesidade promovida pela saciedade $^{41}$. Além disso, as fibras fazem parte do grupo de alimentos funcionais as quais possuem propriedades quimiopreventivas, capazes de barrar o processo de carcinogênese ${ }^{42}$.

Com a transição nutricional que o Brasil vem sofrendo, o sobrepeso e a obesidade aumentaram consideravelmente, aliado a isso, a diminuição do consumo de fibras, o aumento de alimentos embutidos, industrializados e de baixo valor nutricional faz com que cada vez mais doenças como o câncer ganhem espaço ${ }^{45}$. Diversos estudos $33,43,44$ tem demonstrado a correlação da má alimentação e o excesso de peso com o câncer de mama, doença cada vez mais prevalente entre as mulheres pós menopausa ${ }^{46}$.

Conclui-se que o consumo alimentar observado está aquém de ser adequado e o excesso de peso ainda é prevalente nesse grupo de mulheres. Sendo assim, é de extrema importância a elaboração de estratégias de reeducação alimentar, salientando a relevância da alimentação saudável e equilibrada para prevenção e tratamento de doenças, inclusive o câncer de mama, visando a melhora do estado nutricional, manutenção do peso saudável, contribuindo dessa forma para um bom prognóstico.

\section{AGRADECIMENTOS E CONFLITO DE INTERESSE}

Os autores agradecem ao Centro Prudentino de Oncologia (CPO) e as mulheres sobreviventes de câncer de mama em tratamento quimioterápico no referido centro pela viabilização deste trabalho.

Os autores declaram não haver qualquer potencial conflito de interesse que possa interferir na imparcialidade deste trabalho científico.

\section{REFERÊNCIAS}

1. Rodrigues LA, Ligeiro C, Silva M. Cuidados paliativos, diagnósticos e terminalidade: Indicação e início do processo de paliação. CuidArte Enfermagem, 2015 jan-jun; 9(1):26-35.

2. Fernandes AG, Mafra C. Zinco e câncer: uma revisão. Rev. Saúde.com, 2005; 1(2):144-56. Disponível em: http://journaldatabase.info/articles/zinco_cancer _uma_revisao.html

3. Tiezzi DG. Epidemiologia do câncer de mama. Rev Bras Ginecol Obstet. 2009; 31(5):213-5. http://www.scielo.br/pdf/rbgo/v31n5/v31n5a01

4. Instituto Nacional de Câncer José Alencar Gomes da Silva (INCA) Tipos de câncer Mama. Disponível em: http://www2.inca.gov.br/wps/wcm/connect/tipo sdecancer/site/home/mama

5. Instituto Nacional de Câncer José Alencar Gomes da Silva (INCA) Estimativa 2018. Disponível em: http://www1.inca.gov.br/inca/Arquivos/estimativ a-2018.pdf

6. Almeida RA. Impacto da mastectomia na vida da mulher. Revista SBPH2006; 9(2):99-113. Disponível em:http://pepsic.bvsalud.org/scielo.php?script=s ci_arttext\&pid=S1516-08582006000200007

7. Instituto Nacional de Câncer José Alencar Gomes da Silva (INCA). Fatores de risco. Tipos de Câncer. Mama. [citado 2018 dez 8]. Disponível em:

http://www2.inca.gov.br/wps/wcm/connect/tipo sdecancer/site/home/mama/fatores_de_risco_1

8. Irala $\mathrm{CH}$. Qualidade de vida, resposta imune e consumo alimentar de pacientes com câncer de mama do Hospital Universitário de Brasília, 2011. Disponível em: http://repositorio.unb.br/bitstream/10482/1040 3/3/2011_ClarissaHoffmanlrala.pdf 
9. Inumaru LE, Silveira EA, Naves MMV. Fatores de risco e de proteção para câncer de mama: uma revisão sistemática. Cad. Saúde Pública. 2011;

http://doi.org/10.1590/S0102311X2011000700002. 27(7):1259-70.

10. Liz S Qualidade da dieta de mulheres em tratamento adjuvante para o câncer de mama submetidas a um programa de intervenção nutricional educativa [dissertação]. Florianópolis: Universidade Federal de Santa Catarina, Centro de Ciências da Saúde; 2015. Disponível em: https://repositorio.ufsc.br/handle/123456789/16 0782

11. Oliveira DR, Carvalho ESC, Campos LC, Leal JA, Sampaio EV, Cassali GD. Avaliação nutricional de pacientes com câncer de mama atendidas no Serviço de Mastologia do Hospital das Clínicas, Belo Horizonte (MG), Brasil. Rev. Ciên. Sáude Coletiva. 2014; 19(5):1573-80. http://doi.org/10.1590/1413-

$\underline{81232014195.02262013}$

12. Amaral $P$, Miguel $R$, Mehdad A, Cruz C, Monteiro Grillo I, Camilo M, Ravasco P. Body fat and poor diet in breast cancer women. Rev. Nutr Hosp. $2010 \quad$ May-Jun;25(3):456-61. https://www.ncbi.nlm.nih.gov/pubmed/2059313 0

13. Instituto Nacional de Câncer José Alencar Gomes da Silva (INCA) (BRASIL). Sumário Executivo. Políticas e Ações para Prevenção do Câncer no Brasil. Alimentação, Nutrição e Atividade Física. [citado 2018 dez. 8]; Disponível em:

http://bvsms.saude.gov.br/bvs/publicacoes/politi cas_acoes_prevencao_cancer_brasil.pdf

14. Kostner K, Denzer N, Muller C, Klein R, Tilgen W, Reichert J. Den Hollander P, Savage MI, Brown $\mathrm{PH}$. Targeted Therapy for Breast Cancer Prevention. Front Oncol. 2013 Sep 23; 3:250. Review.

https://doi.org/10.3389/fonc.2013.00250

15. Anothaisintawee $\mathrm{T}$, Wiratapun $\mathrm{C}$, Lerdsitthichai P, Kasamesup V, Wongwaisayawan $S$, Srinakarin J, Hirunpat S, Woodtichartpreecha P, Boonlikit S, Teerawattananon Y, Thakkinstian A. Risk Factors of Breast Cancer: A Systematic Review and Meta-Analysis. Ásia Pacífico J Public Colloq Vitae 2020 set-dez; 12(3): 26-36.

Artigo Open Access sob uma licença CC BY-NC-ND (http://creativecommons.org/licenses/by-nc-nd/4.0/).
Health. 2013; 25(5): 368-87. https://doi.org/10.1177/1010539513488795

16. Wolin KY, Carson K, Colditz GA. Obesity and cancer. Oncologist 2010; 15(6):556-65. https://doi.org/10.1634/theoncologist.2009-0285

17. Instituto Nacional de Câncer José Alencar Gomes da Silva (INCA) Prevenção. Câncer de Mama. [citado 2018 dez. 8]. Disponível em: https://www.inca.gov.br/tipos-de-cancer/cancerde-mama

18. Anjos JC, Hofelmann DA. Consumo alimentar e câncer de mama em mulheres de Joinville. Um estudo de Caso-Controle.Revista Brasileira de Cancerologia 2011 [citado 2018 nov.; 57(2): 17787.

Disponível em: https://rbc.inca.gov.br/site/arquivos/n_57/v02/p df/05_artigo_consumo_alimentar_cancer_mama _mulheres_joinville_estudo_caso_controle.pdf

19. Associação Médica Brasileira. Conselho Federal de Medicina (AMB) Diagnóstico e tratamento do câncer de mama. [citato 2018 nov. 20]. Disponível em: http://www.bibliomed.com.br/diretrizes/pdf/can cer_mama.pdf

20. Zanchin FCF, Siviero J, Santos JS, Silva ACP, Rombaldi RL. Estado nutricional e consumo alimentar de mulheres com câncer de mama atendidas em um serviço de mastologia no interior do Rio Grande do Sul, Brasil. Rev HCPA 2011; 31(3): 336-44. Disponível em: https://seer.ufrgs.br/hcpa/article/view/14728

21. Siqueira SL, Kruse MHL. Agrotóxicos e saúde humana: contribuição dos profissionais do campo da saúde. Rev Esc Enferm USP. 2008; 42(3):584$90 . \quad$ https://doi.org/10.1590/S0080$\underline{62342008000300024}$

22. Chlebowski RT, Aiello E, McTiernan A. Weight loss in breast cancer patient management. J Clin Oncol, 2002; 20:1128-43. https://doi.org/10.1200/JCO.2002.20.4.1128

23. World Health Organization (WHO). Obesity Preventing and managing the global epidemic: report of a WHO Consultation on Obesity. Geneva: WHO; 1998. Disponível em: 
https://www.who.int/nutrition/publications/obes ity/WHO_TRS_894/en/

24. Lipschitz DA. Screening for nutritional status in the elderly. Prim Care. 1994; 22:55-67. Disponível em: https://www.ncbi.nlm.nih.gov/pubmed/8197257

25. Kamimura $M A$, Baxman $A$, Sampaio $L R$, Cuppari L. Avaliação nutricional. In: Cuppari L, Nutrição clínica no adulto. 3a. ed. São Paulo: Manole; 2014. p. 89-115.

26. Mussoi TD. Avaliação nutricional na prática clínica: da gestação ao envelhecimento. Rio de Janeiro: Guanabara Koogan; 2015.

27. Sociedade Brasileira de Pediatria. [citado 2019 nov. 11]. Disponível em: https://www.sbp.com.br/fileadmin/user_upload/ img/documentos/valores_referencia.pdf

28. Padovani RM, Amaya-Farfán J, Colugnati FAB, Domene SMA. Dietary reference intakes: aplicabilidade das tabelas em estudos nutricionais (DRI). Rev. Nutr., Campinas, 2006; 19(6):741-60. https://doi.org/10.1590/S1415$\underline{52732006000600010}$

29. Ferreira IB, Marinho EC, Custódio IDD, Gontijo CA, Paiva CE, Crispim CA et al. Consumo alimentar e estado nutricional de mulheres em quimioterapia. Ciência \& Saúde Coletiva, 2016; 21(7):2209-18. https://doi.org/10.1590/1413$\underline{81232015217.05412015}$

30. Leite FMC, Bubach S, Amorim MHC, Castro DS Primo CC. Mulheres com diagnóstico de câncer de mama em tratamento com tamoxifeno: perfil sociodemográfico e clínico. Revista Brasileira de Cancerologia 2011; 57(1): 15-21. Disponível em: http://www1.inca.gov.br/rbc/n_57/v01/pdf/04_a rtigo_mulheres_diagnostico_cancer_mama_trata mento_tamoxifeno.pdf

31. Papa AM, Pirfo CBL, Murad AM, Ribeiro GMQ, Fagundes TC.Impacto da obesidade no prognóstico do câncer de mama. Revista Brasileira de Oncologia Clínica, Belo Horizonte 2013; 9(31):25-30. Disponivel em: https://www.sboc.org.br/sboc-site/revistasboc/pdfs/31/artigo3.pdf

32. Figueiredo ACDS, Ferreira RNF, Duarte $M A G$, Coelho AF, Assis C, Kathryn MA. Prevalência da obesidade em mulheres tratadas de câncer de Colloq Vitae 2020 set-dez; 12(3): 26-36.

Artigo Open Access sob uma licença CC BY-NC-ND (http://creativecommons.org/licenses/by-nc-nd/4.0/). mama numa UNACOM em Juiz de Fora, Minas Gerais.Rev Bras Mastologia. 2016; 26(4):169-74. https://doi.org/10.5327/Z201600040006RBM

33. Rubin BA, Stein AT, Zelmanowicz AM, Rosa DD. Perfil Antropométrico e Conhecimento Nutricional de Mulheres Sobreviventes de Câncer de Mama do Sul do Brasil. Revista Brasileira de Cancerologia 2010; 56(3): 303-9. http://www1.inca.gov.br/rbc/n_56/v03/pdf/03_a rtigo_perfil_antropometrico_conhecimento_nutri cional_mulheres_cancer_mama_sul.pdf

34. Lagares EB, Santos KF, Mendes RC, Moreira FA, Anastácio LR. Excesso de Peso em Mulheres com Diagnóstico de Câncer de Mama em Hormonioterapia com Tamoxifeno. Revista Brasileira de Cancerologia 2013; 59(2): 201-10. Disponível em: https://rbc.inca.gov.br/site/arquivos/n_59/v02/p df/07-excesso-de-peso-em-mulheres-comdiagnostico-de-cancer-de-mama-emhormonioterapia-com-tamoxifeno.pdf

35. Instituto Nacional de Câncer José Alencar Gosmes (INCA). Consenso Nacional de Nutrição Oncológica. 2a ed. rev. Rio de Janeiro: INCA, 2016. [citado 2019 nov. 25]. Disponível em: https://www.inca.gov.br/publicacoes/livros/cons enso-nacional-de-nutricao-oncologica

36. Cattafesta $M$, Siqueira JH, Podestá OPG, Podestá JRV, Salaroli LB. Consumo alimentar de pacientes com câncer de mama acompanhados em centro especializado em oncologia na grande vitória/es-brasil. Revista Brasileira de Oncologia Clínica 2014; 10(38):124-31. Disponivel em: http://www.nutricao.ufes.br/sites/nutricao.ufes. br/files/Consumo\%20Alimentar\%20de\%20Pacien tes\%20com\%20C\%C3\%A2ncer.pdf

37. Associação Médica Brasileira (AMB). Diretrizes. Sociedade Brasileira de nutrição parental e enteral; associação brasileira de nutrologia. Terapia nutricional na oncologia, 2011. [citado 2019 nov. 25]. Disponível em: https://diretrizes.amb.org.br/_bibliotecaantiga/t erapia_nutricional_na_oncologia.pdf

38. Instituto Nacional de Câncer José Alencar Gomes da Silva (INCA) Rev. Consenso Nacional de Nutrição Oncológica; Vol. II. Rio de Janeiro, 2016. Disponível em: 
https://www.inca.gov.br/publicacoes/livros/cons enso-nacional-de-nutricao-oncologica

39. Silva CT, Jasiulionis MG. Relação entre estresse oxidativo, alterações epigenéticas e câncer. Cienc. Cult. 2014; 66(1):38-42.. https://doi.org/10.21800/S0009-

\section{$\underline{67252014000100015}$}

40. Rios AO, Antunes LMG, Bianchi MLP. Proteção de carotenoides contra radicais livres gerados no tratamento de câncer com cisplatina. Alim. Nutr., Araraquara 2009; 20(2):343-50. Disponivel em: http://serv-

bib.fcfar.unesp.br/seer/index.php/alimentos/arti cle/viewFile/1067/818

41. Catalani LA et al. Fibras Alimentares. Rev Bras Nutr Clin 2003; 18(4):178-82.

42. Padilha PC, Pinheiro RL. O Papel dos Alimentos Funcionais na Prevenção e Controle do Câncer de Mama. Revista Brasileira de Cancerologia 2004; 50(3): 251-60. Disponível em: http://www1.inca.gov.br/rbc/n_50/v03/pdf/REVI SAO3.pdf

43. Mota JCM, Martins KA, Mota JF, FreitasJunior, R. Excesso de peso e de gordura androide em mulheres goianas recém-diagnosticadas com câncer de mama. Rev Bras Mastologia. 2016; 26(2):50-5.

https://doi.org/10.5327/Z201600020004RBM

44. Felden JBB, Figueiredo ACL. Distribuição da gordura corporal e câncer de mama: um estudo de caso-controle no Sul do Brasil. Ciênc. saúde coletiva 2011; 16(5): 2425-33. https://doi.org/10.1590/S1413-81232011000500011. https://doi.org/10.1590/S1413-

\section{$\underline{81232011000500011}$}

45. Ambrosio C, Di Pietro PF, Rockenbach G, Vieira FGK, Galvan D, Crippa CG. et al. Fatores que influenciam o consumo energético de mulheres no tratamento do câncer de mama. Bras. Ginecol. Obstet. $2011 \quad$ Ago 33(8):207-13. https://doi.org/10.1590/S0100-

$\underline{72032011000800007}$

46. Matos JC, Pelloso SM, Carvalho MDB Prevalência de fatores de risco para o câncer de mama no município de Maringá, Paraná. Revista
Latino-Americana de Enfermagem 2010; 18(3):352-9. https://doi.org/10.1590/S0104$\underline{11692010000300009}$ 